J. Austral. Math. Soc. 20 (Series A) (1975), 301-304.

\title{
A CLASS OF HARMONICALLY CONVERGENT SETS
}

\author{
TORLEIV KLØVE
}

Communicated by B. Mond

(Received 17 April 1974)

\section{1.}

Following Craven (1965) we say that a set $M$ of natural numbers is harmonically convergent if

$$
\mu(M)=\sum_{n \in M} \frac{1}{n}
$$

converges, and we call $\mu(M)$ the harmonic sum of $M$. (Craven defined these concepts for sequences rather than sets, but we found it convenient to work with sets.) Throughout this paper, lower case italics denote non-negative integers.

Let $r>1,1 \leqq m \leqq r$, and $0 \leqq d_{1}<d_{2}<\cdots<d_{m}<r$. We define

$$
M(t)=M\left(r ; d_{1}, d_{2}, \cdots, d_{m} ; t_{1}, t_{2}, \cdots, t_{m}\right)
$$

to be the set of natural numbers which contains the digit $d_{i}$ exactly $t_{i}$ times $(i=1,2, \cdots, m)$ when expressed in the scale of $r$. Further, let

$$
\begin{aligned}
M_{\lambda}(t) & =\left\{\left[n^{\lambda}\right] \mid n \in M(t)\right\}, \\
M_{\lambda}^{0}(t) & =\left\{\left[n^{i}\right] \mid n \in M(t), n<r\right\}, \\
T & =\sum_{i=1}^{m} t_{i}, \\
T^{*} & =\prod_{i=1}^{m}\left(t_{i} !\right),
\end{aligned}
$$

where $[x]$ denote the least integer $\geqq x$ and $0 !=1$. Note that $M_{\lambda}^{0}(t)$ is empty if $T>1$. We prove the following theorems. 
THEOREM 1. If $d_{1}>0$ and $\lambda>\log (r-m) / \log r$, then

$$
\begin{aligned}
\mu\left(M_{\lambda}(t)\right) \leqq \mu\left(M_{\lambda}^{0}(t)\right)+\frac{T ! r^{\lambda}}{T^{*}\left(r^{\lambda}-r+m\right)^{T+1}} & \left(\sum_{n=1}^{r-1} \frac{1}{n^{\lambda}}-\sum_{i=1}^{m} \frac{1}{d_{i}^{\lambda}}\right) \\
& +\frac{(T-1) ! r^{\lambda}}{T^{*}\left(r^{\lambda}-r+m\right)^{T}} \sum_{i=1}^{m} \frac{t_{i}}{d_{i}^{\lambda}} .
\end{aligned}
$$

THEOREM 2. If $d_{1}>0, m<r$, and $0 \leqq \lambda \leqq \log (r-m) / \log r$, then $M_{\lambda}(t)$ is not harmonically convergent, i.e. $\mu\left(M_{\lambda}(t)\right)=\infty$.

2.

To prove the theorems, we first prove some lemmata. We assume throughout that $d_{1}>0$.

LEMMA 1 . For $l \geqq 1$ we have

$$
\begin{aligned}
& \sum_{\substack{o \leqq b<r^{l} \\
b \in M(l)}} 1=\frac{l !(r-m)^{l-T}}{T^{*}(l-T) !} \text { if } l \geqq T, \\
& =0 \quad \text { if } l<T .
\end{aligned}
$$

Proof. The case $l<T$ is obvious. Let $l \geqq T$. If $b<r^{l}$ then

$$
b=\sum_{j=0}^{l-1} b_{j} r^{j} \text { where } 0 \leqq b_{j}<r
$$

The sum in the lemma equals the number of ways we may choose $\left(b_{0}, b_{1}, \cdots, b_{l-1}\right)$ such that $b_{j}=d_{i}$ for exactly $t_{i}$ values of $j(i=1,2, \cdots, m)$. The $T$ element of which $t_{i}$ have value $d_{i}$ may be chosen in

$$
\frac{l !}{t_{1} ! t_{2} ! \cdots t_{m} !(l-T) !}
$$

ways, and for the remaining $(l-T)$ elements there are $(r-m)$ possible choices. This proves the lemma.

LEMMA 2. For $\lambda>\log (r-m) / \log r$ we have

$$
\sum_{l=1}^{\infty} \frac{1}{r^{\lambda l}} \sum_{\substack{0 \leqq b<r \\ b \in M(l)}} 1=\frac{T ! r^{\lambda}}{T^{*}\left(r^{\lambda}-r+m\right)^{T+1}} .
$$

Proof. By Lemma 1 we get

$$
\sum_{l=1}^{\infty} \frac{1}{r^{\lambda l}} \sum_{\substack{0 \leq b<r l \\ b \in M(t)}} 1=\sum_{l=1}^{\infty} \frac{1}{r^{\lambda l}} \cdot \frac{l !(r-m)^{l-T}}{T^{*}(l-T) !}
$$




$$
\begin{aligned}
& =\frac{T !}{T^{*}(r-m)^{T}} \sum_{l=T}^{\infty}\left(\begin{array}{c}
l \\
T
\end{array}\right)\left(\frac{r-m}{r^{\lambda}}\right)^{l} \\
& =\frac{T !}{T^{*}(r-m)^{T}} \cdot \frac{\left(\frac{r-m}{r^{\lambda}}\right)^{T}}{\left(1-\frac{r-m}{r^{\lambda}}\right)^{T+1}} \\
& =\frac{T ! r^{\lambda}}{T^{*}\left(r^{\lambda}-r+m\right)^{T+1}} \cdot
\end{aligned}
$$

We now prove Theorem 1 . For $k \geqq 1$ and $\lambda>\log (r-m) / \log r$ we have, with $t_{i}=\left(t_{1}, \cdots, t_{i-1}, t_{i}-1, t_{i+1}, \cdots, t_{m}\right)$, and $\Delta=\left\{a \mid 1 \leqq a<r\right.$ and $a \neq d_{i}$ for $i=1,2, \cdots, m\}$,

$$
\begin{aligned}
& \sum_{\substack{1 \leqq n<r^{k+1} \\
n \in M(t)}} \frac{1}{\left[n^{\lambda}\right]} \leqq \sum_{\substack{1 \leqq n<r \\
n \in M(t)}} \frac{1}{\left[n^{\lambda}\right]}+\sum_{l=1}^{k} \sum_{r^{l} \leqq n<r^{l+1}} \frac{1}{n^{\lambda}} \\
& \quad=\mu\left(M_{\lambda}^{0}(t)\right)+\sum_{l=1}^{k}\left\{\sum_{a \in \Delta} \sum_{\substack{0 \leqq b<r^{l} \\
b \in M(t)}} \frac{1}{\left(a r^{l}+b\right)^{\lambda}}+\sum_{i=1}^{m} \sum_{\substack{0 \leqq b<r^{l} \\
b \in M\left(t_{i}\right)}} \frac{1}{\left(d_{i} r^{l}+b\right)^{\lambda}}\right\} \\
& \quad \leqq \mu\left(M_{\lambda}^{0}(t)\right)+\sum_{a \in \Delta} \frac{1}{a^{\lambda}} \sum_{\substack{l=1 \\
l=1}}^{k} \frac{1}{r^{l \lambda}} \sum_{\substack{0 \leqq b<r^{l} \\
b \in M(t)}}^{\sum}+\sum_{i=1}^{m} \frac{1}{d_{i}^{\lambda}} \sum_{l=1}^{k} \frac{1}{r^{l \lambda}} \sum_{\substack{0 \leqq b<r^{l} \\
b \in M\left(t_{i}\right)}} 1 .
\end{aligned}
$$

Letting $k \rightarrow \infty$, Theorem 1 follows from this by Lemma 2 .

To prove Theorem 2, we first note that $[x]<2 x$ for $x>1$. Hence, by Lemma 1,

$$
\begin{aligned}
& \sum_{\substack{1 \leqq n<r^{k+1} \\
n \in M(s)}} \frac{1}{\left[n^{\lambda}\right]}>\frac{1}{2} \sum_{\substack{l=1 \\
l=1}} \sum_{\substack{r^{l} \leqq n<r^{l+1} \\
n \in M(l)}} \frac{1}{n^{\lambda}}>\frac{1}{2} \sum_{l=1}^{k} \frac{1}{r^{\lambda(l+1)}} \sum_{\substack{r^{l} \leqq n<r^{l+1} \\
n \in M(t)}} 1 \\
& =\frac{1}{2} \sum_{l=T}^{k} \frac{1}{r^{\lambda(l+1)}}\left\{\frac{(l+1) !(r-m)^{l+1-T}}{T^{*}(l+1-T) !}-\frac{l !(r-m)^{l-T}}{T^{*}(l-T) !}\right\} \\
& \geqq \frac{1}{2} \sum_{l=T}^{k} \frac{T !}{T^{*}(r-m)^{T} r^{\lambda}}\left\{\left(\begin{array}{c}
l+1 \\
T
\end{array}\right)\left(\frac{r-m}{r^{\lambda}}\right)^{l+1}-\left(\begin{array}{c}
l \\
T
\end{array}\right)\left(\frac{r-m}{r^{\lambda}}\right)^{l}\right\} \\
& =\frac{T !}{2 T^{*}(r-m)^{T} r^{\lambda}}\left\{\left(\begin{array}{c}
k+1 \\
T
\end{array}\right)\left(\frac{r-m}{r^{\lambda}}\right)^{k+1}-\left(\frac{r-m}{r^{\lambda}}\right)^{T}\right\} \rightarrow \infty
\end{aligned}
$$

when $k \rightarrow \infty$. 
3.

In Kløve (1971) we treated the case: $\lambda$ integer, $d_{1}>0$, and $t_{i}=0, i=1,2, \cdots, m$. The estimates given there are better then those given by Theorem 1 . Better estimates may be given in the general case, but the expressions seem to be very complicated.

Craven (1965) and Alexander (1971) gave estimates for $\mu\left(\bigcup_{t=0}^{T} M(t)\right)$ in the special case $m=1$. Improved estimates for this sum may be obtained from Theorem 1 . In general, if $M_{j}, j=1,2, \cdots, s$ are harmonically convergent sets, then so is $\bigcup_{j=1}^{s} M_{j}$ and

$$
\mu\left(\bigcup_{j=1}^{s} M_{j}\right) \leqq \sum_{j=1}^{s} \mu\left(M_{j}\right)
$$

In fact, $\mu$ is a measure on the $\sigma$-algebra of all subsets of the set of natural numbers. If we consider the special case $\lambda=m=1$ in Theorem 1 , we get, for $t>0$,

Further

$$
\begin{aligned}
\mu(M(t)) & \leqq \mu\left(M_{1}^{0}(t)\right)+r\left(\sum_{n=1}^{r-1} \frac{1}{n}-\frac{1}{d}\right)+\frac{r}{d} \\
& \leqq \mu\left(M_{1}^{0}(t)\right)+r(\log r+1) .
\end{aligned}
$$

$$
\begin{aligned}
\mu(M(0)) & \leqq r(\log r+1), \\
\mu\left(M_{1}^{0}(t)\right) & =\frac{1}{d} \text { if } t=1 . \\
& =0 \text { if } t>1 .
\end{aligned}
$$

Hence, for $T \geqq 1$,

$$
\mu\left(\bigcup_{t=0}^{T} M(t)\right) \leqq(T+1) r \log r+(T+1) r+\frac{1}{d} .
$$

\section{References}

R. Alexander (1971), 'Remarks about the digits of integers', J. Austral. Math. Soc. 12, 239-241.

B. D. Craven (1965), 'On digital distribution in some integer sequences', J. Austral. Math. Soc. 5 325-330.

T. Kløve (1971), 'Power sums of integers with missing digits', Math Scand. 28, 247-251.

University of Bergen,

Bergen, Norway. 